\title{
Activos del Barrio y Ajuste Adolescente
}

\section{Neighborhood Assets and Adolescent Adjustment}

\author{
Alfredo Oliva Delgado, Lucía Antolín Suárez, Rosa Ma Estévez Campos y Diana Mª Pascual García \\ Universidad de Sevilla, España
}

\begin{abstract}
Resumen. En este estudio se analiza la relación entre algunas dimensiones o activos para el desarrollo en el barrio de residencia, medidos mediante la Escala para la Evaluación de los Activos del Barrio, y el ajuste y la satisfacción vital de adolescentes. La muestra estuvo formada por 2400 adolescentes (1068 chicos y 1332 chicas) con edades comprendidas entre los 12 y los 17 años, que cursaban estudios de Educación Secundaria en centros de Andalucía Occidental. Los resultados mostraron relaciones significativas entre la mayoría de las dimensiones de la escala (empoderamiento de la juventud, apego al barrio, seguridad y control social) y los problemas internalizantes y externalizantes, el consumo de sustancias y la satisfacción vital de los participantes. Por otra parte, los datos aportan evidencias acerca de la validez externa de la escala utilizada. A partir de los resultados se ofrecen algunas sugerencias para la intervención en el entorno comunitario.

Palabras clave: ajuste adolescente, barrio, satisfacción vital, validación de escala.
\end{abstract}

\begin{abstract}
This paper presents the results of a study analyzing the relationship between some dimensions or developmental assets in the neighborhood, as measured by the Scale for the Assessment of Developmental Assets in the Neighborhood, and adjustment and life satisfaction of adolescent boys and girls. The sample consisted of 2400 adolescents (1068 boys and 1332 girls) between ages 12 and 17 who were secondary students in public and private schools in Western Andalusia. The results showed significant relationships between most dimensions of the scale (youth empowerment, attachment to neighborhood safety and social control) and internalizing and externalizing problems, substance use and life satisfaction of participants. Moreover, the data provide evidence about the external validity of the scale used in the study. From the results some suggestions for intervention in the community environment are extracted.
\end{abstract}

Keywords: adolescent adjustment, life satisfaction, neighborhood, scale validation.

El interés por estudiar las influencias que los contextos de desarrollo de niños y adolescentes tienen sobre su ajuste y bienestar psicológico llevó a muchos investigadores a poner el foco en la familia y la escuela, pues, como indicó Bronfrenbrenner (1979), estos son los escenarios en los que transcurre la vida de la mayor parte de niños y niñas. Sin embargo, con la llegada de la adolescencia cada vez es mayor el tiempo que chicos y chicas pasan fuera de la casa y la escuela, lo que supone una mayor exposición a la influencia de factores relacionados con el barrio en que residen (Larson, Richards, Moneta, Holmbeck y Duckett, 1996). Por otra parte, cuando los niños son pequeños las influencias vecinales pueden estar mediadas por variables familiares, pero cuando crecen están más inmersos en redes sociales del vecindario, con lo que aumentan los efectos directos del barrio sobre su conducta (Kroneman, Loeber y Hipwell, 2004).

Como han apuntado algunos autores (Leventhal, Dupèrè y Brooks-Gunn, 2009; Sampson, Morenoff y

Correspondencia: Alfredo Oliva Delgado. Departamento de Psicología Evolutiva y de la Educación. Facultad de Psicología. Universidad de Sevilla. C/ Camilo José Cela, s/n. 41018 Sevilla. E-mail: oliva@us.es
Gannon-Rowley, 2002), los primeros estudios acerca de las influencias que el barrio de residencia ejercen sobre el desarrollo y comportamiento infanto-juvenil tuvieron como objetivo detectar cuáles eran los factores que explicaban la elevada tasa de problemas comportamentales y escolares que se observaban en algunos suburbios de grandes ciudades de Estados Unidos y Europa. Muchos de estos estudios se centraron en una única dimensión del barrio, el nivel socioeconómico de sus residentes y otros factores relacionados con él, como la estabilidad residencial, la heterogeneidad étnica o las tasas de residentes que eran propietarios de sus viviendas. Ello proporcionaba escasa información acerca de los procesos implicados en la relación y dificultaba la extracción de recomendaciones para la intervención comunitaria. Hubo que esperar hasta la última década del pasado siglo para que comenzaran a surgir las primeras explicaciones acerca de los mecanismos y procesos a través de los cuales el barrio podía ejercer su influencia sobre sus residentes de menor edad. Así, Jencks y Mayer (1990) hicieron referencia a la importancia de tener en cuenta los procesos de socialización colectiva, la influencia del grupo o los recursos institucionales disponibles en el barrio. Desde entonces, y a lo largo de las dos últimas décadas, se ha ido acumu- 
lando una abundante evidencia empírica sobre la relación entre factores relativos al barrio y distintos indicadores de desajuste conductual y emocional, tales como el comportamiento antisocial y violento (Haynie, Silver y Teasdale, 2006), el consumo de sustancias (Brody et al., 2001; Ge, Brody, Conger, Simons y Murry, 2002), las conductas sexuales de riesgo (Cleveland y Gilson, 2004; Dupèrè, Lacourse, Willms, Leventhal y Tremblay, 2008), el rendimiento escolar (Boyle, Georgiades, Racine y Mustard, 2007) o los síntomas depresivos (Wickrama, Merten y Elder, 2005; Herrero y Gracia, 2007).

Partiendo de un marco teórico diferente, durante los últimos años el modelo del desarrollo positivo adolescente también ha destacado la importancia de los factores relativos al barrio o vecindario. Así ha propuesto el concepto de activos para el desarrollo para hacer referencia a aquellos recursos personales, familiares, escolares o comunitarios que promueven la competencia y el desarrollo adolescente $y$, a su vez, previenen la aparición de problemas. Entre los activos de barrio algunos autores han citado, la seguridad, la existencia de límites claros o el empoderamiento y la valoración positiva de los jóvenes por parte de la población adulta (Benson, Scales, Hamilton y Sesman, 2006; Oliva et al., 2010).

Entre todas las dimensiones del barrio, la seguridad es una de las que con más frecuencia ha sido tenida en cuenta por los investigadores. Así, la falta de seguridad ha aparecido muchas veces relacionada con el desajuste comportamental de jóvenes y adolescentes. Son muchos los estudios que encuentran que aquellos chicos y chicas que residen en vecindarios en los que no hay fácil acceso a las drogas, y en los que la violencia entre grupos y los actos antisociales son poco frecuentes muestran un mejor ajuste, no sólo conductual sino también emocional (Duncan, Duncan y Strycker, 2002). Por el contrario, la disponibilidad de sustancias ilegales en el vecindario es un importante predictor de su consumo, así como de las conductas violentas y de la afiliación a bandas (Lambert, Brown, Phillips y Ialongo, 2004).

Sampsom et al. (2002), en su revisión de literatura empírica acerca de los efectos del barrio sobre la salud y los problemas de conducta, destacaron la importancia de diversos factores, algunos de ellos relativos al clima social y otros relacionados con los recursos y las rutinas o actividades que estos facilitan o promueven. Entre los primeros se pueden citar las redes sociales existentes y la confianza recíproca entre los vecinos, que facilitan la acción y cooperación para el beneficio mutuo entre los miembros de una comunidad. Ello podría considerarse el capital social del barrio o comunidad vecinal (Halpern, 2005; O'Connor et al., 2011; Putnam, 1993), que algunos estudios han asociado a un menor consumo de sustancias y problemas comportamentales (Calafat et al., 2011; Weitzman y Chen, 2005). Muy relacionado con ello estaría el sentido de comunidad (McMilan y Chavis, 1986), aunque, como han señalado Cantillon, Davidson y Schweitzer (2003), este sentido de comunidad puede resultar algo difuso e incluir componentes o dimensiones más concretas, tales como la seguridad, el apoyo y empoderamiento percibidos, o la vinculación al vecindario (Chipuer y Pretty, 1999; Zani, Cicognani y Albanesi, 2001). Uno de los rasgos esenciales de este sentido de comunidad sería el apego o la fuerte vinculación emocional con el barrio, y que supone un sentimiento de que el residente se preocupa por su vecindario y el vecindario se preocupa por sus residentes (Cantillon et al., 2003). Algunos estudios con adultos han encontrado relaciones positivas entre la vinculación o integración al barrio de residencia y el bienestar psicológico y la salud (Cohen, Gottlieb y Underwood, 2000; Gracia y Herrero, 2004).

Este sentimiento de apego al barrio aumentaría el deseo de implicarse de forma activa en beneficio de la comunidad, algo que ha sido definido por algunos autores como eficacia colectiva (Cancino, 2005), y que para Leventhal y Brooks-Gunn (2000) es uno de los principales mecanismos mediante los que el vecindario puede ejercer su influencia. Esta implicación redundaría en un mayor interés de las personas del vecindario por controlar y supervisar el comportamiento de niños y adolescentes y su disposición a intervenir en caso de detectar conductas incívicas y contrarias a las normas de la comunidad vecinal. Así pues, este control social puede considerarse otro importante recurso del barrio, que sería el equivalente comunitario del control o supervisión parental, y que, al igual que éste, está relacionado con la prevención de comportamientos antisociales (Jessor y Jessor, 1977; Sampson y Groves, 1989).

Aunque no existe evidencia empírica al respecto, el modelo de desarrollo positivo adolescente también ha destacado la importancia de la valoración positiva y la asignación de responsabilidades y roles a jóvenes y adolescentes por parte de la comunidad (Benson et al., 2006). De acuerdo con estos autores, una visión favorable y una actitud de apoyo y empoderamiento puede contribuir de forma decisiva a que los jóvenes maduren, se sientan útiles e importantes para la comunidad y quieran contribuir de forma activa a su mejora, lo que llevaría a un mejor ajuste psicológico.

Sampson et al. (2002) habían apuntado la importancia de las rutinas y actividades, algo que resulta de especial relevancia en el caso de jóvenes y adolescentes. Así, una importante evidencia empírica indica que la existencia de actividades extraescolares o de ocio estructuradas a las que los adolescentes puedan dedicar su tiempo libre también está relacionada con la competencia académica y con el desarrollo personal y social. Cada vez disponemos de más estudios que destacan la importancia de la implicación en este tipo de actividades para la formación integral de la juventud y para la reducción de muchos problemas conductuales, espe- 
cialmente en jóvenes en situación de riesgo psicosocial (Eccles y Gootman, 2002; Gardner, Roth y BrooksGunn, 2008; Mahoney y Stattin, 2000). Por lo tanto, se trata de uno de los activos del barrio que pueden considerarse más relevantes para fomentar el ajuste y el bienestar adolescente.

La mayoría de estudios que vinculan las características del barrio con el desarrollo y ajuste adolescente se basan en medidas objetivas y estructurales, a las que se tiene fácil acceso a través del censo (tales como el nivel educativo o socioeconómico de sus residentes), o de determinadas fuentes de información como profesionales de los servicios sociales, sanitarios o educativos del distrito. Sin embargo, aunque este enfoque puede ser interesante para conocer los recursos o actividades para jóvenes disponibles en el barrio, no resulta muy eficaz para captar otras dimensiones, como el sentimiento de apego al barrio o la sensación de seguridad. Por ello, la valoración que los adolescentes hacen de su propio vecindario es una fuente de información muy útil de cara a determinar la asociación existente entre algunas de las características del barrio y el ajuste y desarrollo de sus residentes jóvenes (Burton y Jarret, 2000; Gracia, Fuentes y García, 2010). Por otra parte, no faltan estudios que encuentran que las percepciones que los adolescentes tienen de su barrio están más relacionadas con su ajuste que las medidas objetivas (Plunkett, Abarca-Mortersen, Behnke y Sands, 2007), probablemente porque estas percepciones ejercen una función mediadora entre las condiciones reales del vecindario y la salud y el ajuste de sus residentes (Roosa, White, Zeiders y Tein, 2009).

Aunque existen algunas escalas para valorar la percepción que los residentes tienen de su barrio o vecindario, no suelen estar pensadas para medir sus bondades como contextos favorecedores del desarrollo y ajuste adolescente, pues fueron diseñadas pensando en personas adultas o niños y niñas de menos edad (Herrero y Gracia, 2007; Martínez, Black y Starr, 2002; Peterson, Speer y McMillan, 2007). En este estudio se ha utilizado una escala que evalúa la percepción de los chicos y chicas acerca de diferentes recursos o activos del barrio en el que residen, por lo que puede aportar una información muy interesante acerca de los activos comunitarios que podrían promoverse de cara a favorecer el ajuste y el bienestar adolescente.

Por otra parte, estos datos también servirán para aportar sobre la validez externa de la escala empleada, que ya ha mostrado una excelente validez de constructo (Oliva, Antolín y López, en prensa). Un aspecto importante de este estudio es la inclusión de varios indicadores de ajuste emocional adolescente, los problemas internalizantes y la satisfación vital, que van más allá de los clásicos problemas comportamentales o delictivos que suelen utilizarse en muchos estudios.

Por lo tanto, los objetivos del estudio son dos aunque estrechamente relacionados. En primer lugar, ana- lizar la relación de distintas dimensiones o activos del barrio con los problemas internalizantes y externalizantes, con el consumo de sustancias y con la satisfacción vital de sus residentes adolescentes, que puede considerarse un buen indicador de bienestar psicológico y desarrollo positivo adolescente. En segundo lugar, aportar datos acerca de la validez externa de la escala empleada para evaluar estos activos del barrio, que unidos a la excelente validez de constructo ya demostrada (Oliva et al., en prensa), justifiquen la utilización de la escala con fines de investigación o intervención.

Nuestras hipótesis de partida fueron que las percepciones favorables de los adolescentes sobre dimensiones del barrio como el empoderamiento juvenil, el apego al barrio, su seguridad, el control social, o la disponibilidad de actividades de ocio, estarán asociadas a un mejor ajuste interno y externo y a una mayor satisfacción vital.

\section{Método}

\section{Sujetos}

La muestra seleccionada estuvo constituida por 2400 adolescentes (1068 chicos y 1332 chicas) de 12 a 17 años $(M=14.73, D T=1.25)$ que cursaban estudios de Educación Secundaria en centros públicos y privados de Andalucía Occidental. Todos ellos fueron seleccionados en 20 centros educativos elegidos en función del: a) tamaño del centro (pequeño: menos de 600 alumno/as, grande: más de 600), b) nivel socioeconómico de la zona (media-baja y media-alta), c) tamaño de la población donde se sitúa (pequeño: menos de 30.000 habitantes, grande: más de 30.000) y d) titularidad (público o privado). En cada centro fueron seleccionadas al azar dos aulas de cada uno de los cursos y niveles educativos de $2^{\circ}, 3^{\circ}$ y $4^{\circ}$ de E.S.O., $1^{\circ}$ de Bachillerato y $1^{\circ}$ de Ciclos Formativos de Grado Medio. Sólo el alumnado menor de 18 años fue seleccionado para formar parte de la muestra.

\section{Instrumentos}

Escala para la Evaluación de los Activos del Barrio (Oliva et al., en prensa). Se trata de una escala tipo Likert compuesta por 22 ítems que permite evaluar la percepción que chicos y chicas adolescentes tienen de diferentes factores del vecindario en el que residen, y que pueden promover su ajuste y desarrollo psicológico. La escala cuenta con importantes evidencias de validez, mostrando unos buenos índices de ajuste y una estructura de cinco dimensiones, que son las siguientes:

- Apoyo y Empoderamiento de la juventud: Se refiere al grado en que los adolescentes perciben que las personas adultas de su barrio se preocupan 
por sus jóvenes, los apoyan, los valoran y fomentan su participación. Se trata, por lo tanto, de una dimensión que incluye contenidos que, aunque pudieran considerarse diferenciados a priori, agrupó el análisis factorial confirmatorio realizado para la validación de la escala. La etiqueta de apoyo y empoderamiento define bien sus contenidos. Esta compuesta por seis ítems (por ejemplo, "la gente adulta de mi barrio valora mucho a los jóvenes"). El coeficiente de fiabilidad, alfa de Cronbach, para la subescala fue de .91 .

- Apego al barrio: Incluye cuatro ítems referidos al sentimiento de pertenencia o vinculación con la barriada ("Siento que formo parte de mi barrio"). Alfa de Cronbach = .91.

- Seguridad del barrio: Esta subescala está formada por cuatro ítems a través de los cuales quien cumplimenta el cuestionario indica la percepción que tiene de la seguridad de su barrio y la ausencia de delitos y violencia ("La gente de mi barrio comete delitos y gamberradas"). Obtuvo una fiabilidad de alfa de Cronbach de .87.

- Control Social: Se refiere a la supervisión y control que las personas adultas del vecindario ejercen sobre jóvenes y adolescentes, y a su predisposición a intervenir en caso de que observen comportamientos inapropiados. Está formada por cuatro ítems ("En mi barrio si haces cualquier gamberrada seguro que algún adulto te reñirá"). Alfa de Cronbach $=.85$.

- Actividades para jóvenes: Los cuatro ítems que conforman esta dimensión están referidos a la oferta de actividades de ocio dirigidas a las personas jóvenes que se llevan a cabo en el barrio ("Hay pocos barrios en los que haya tantas actividades para jóvenes como en el mío"). Obtuvo una fiabilidad de alfa de Cronbach de .80 .

En todas las subescalas las puntuaciones altas indicaron aspectos positivos o favorables del barrio.

Nivel Socio-económico. Fue utilizada la Revised Family Afluence Scale (Boyce, Torsheim, Currie y Zamborn, 2006), que construye un índice a partir de preguntas referidas al número de coches y ordenadores que posee la familia, la existencia de habitación propia para el adolescente, o los desplazamientos realizados durante las vacaciones. El rango de este índice estaba comprendido entre 0 y $9(M=5.75, D T=1.97)$.

Youth Self Report (YSR; Achenbach, 1991). Se trata de una escala compuesta por 100 ítems y diseñada para ser utilizada con adolescentes de edades comprendidas entre los 12 y los 18 años. Se empleó la versión castellana de Lemos, Vallejo y Sandoval (2002). Todos los ítems deben ser respondidos eligiendo entre tres opciones: 0 "nada verdadero", 1 "algo verdadero" y 2 "muy verdadero". Incluye dos grandes dimensiones, una referida a problemas internalizantes o emocionales (depresión, quejas somáticas, problemas de relación) y otra a problemas externalizantes o conductuales (con- ducta delictiva, agresividad verbal, búsqueda de atención). Se obtuvo una fiabilidad de alfa de Cronbach de .80 tanto en la subescala de problemas internalizantes como en la de problemas externalizantes.

Consumo de sustancias. Cuestionario elaborado ad hoc para esta investigación que incluía cuatro preguntas referidas al consumo de tabaco, hachís y alcohol, y a las borracheras experimentadas. Los adolescentes debían señalar el nivel de consumo en una escala comprendida entre 1 "nunca" y 4 "más de cinco veces", en el caso del consumo de hachís y las borracheras, y entre 1 y 5, para el consumo de alcohol "a diario" o tabaco "más de tres cigarrillos diarios". La fiabilidad según el alfa de Cronbach fue de .82 .

Escala para la Evaluación de la Satisfacción Vital. La Student's Life Satisfaction Scale fue elaborada por Huebner (1991). Es un instrumento que sirve para evaluar la satisfacción vital de los chicos y chicas adolescentes, y cuya versión española ha sido validada recientemente (Galíndez y Casas, 2010). Está compuesta por siete ítems (e.g., "tengo lo que quiero en la vida") que deben ser puntuados en una escala tipo Likert comprendida entre 1 (totalmente en desacuerdo) y 7 (totalmente de acuerdo). No dispone de subescalas, por lo que contempla tan sólo una puntuación global. El coeficiente de fiabilidad, alfa de Cronbach, para esta escala fue de .81

\section{Procedimiento}

Los objetivos del estudio fueron explicados al director/a de los centros elegidos en contactos previos, después de los cuales, dos miembros del equipo de investigación los visitaron y aplicaron los instrumentos en las aulas elegidas, que fueron las correspondientes a los niveles de ESO (salvo $1^{\text {er }}$ curso), Bachillerato y Ciclos Formativos, descritos líneas atrás. Sus padres y madres fueron informados por correo del estudio, y ninguno se opuso al mismo. Para garantizar la sinceridad y privacidad de las respuestas, el alumnado de cada una de las clases seleccionadas cumplimentó el cuestionario de forma voluntaria y anónima. Lo hicieron en una sesión de una hora de duración en presencia de un miembro del equipo investigador.

\section{Resultados}

En la Tabla 1 se presentan las correlaciones entre todas las variables incluidas en el estudio. En primer lugar, hay que destacar que aparecieron muchas correlaciones significativas entre las variables referidas al ajuste y bienestar adolescente y las dimensiones del barrio o vecindario. Mientras que tanto los problemas internalizantes como la satisfacción vital se asociaron con todas las variables del barrio, las variables comportamentales sólo mostraron relaciones significativas 
Tabla 1. Correlaciones entre las variables contempladas en el estudio

\begin{tabular}{|c|c|c|c|c|c|c|c|c|c|c|c|c|}
\hline Variables & 1 & 2 & 3 & 4 & 5 & 6 & 7 & 8 & 9 & 10 & 11 & 12 \\
\hline 1. Edad & - & & & & & & & & & & & \\
\hline 2. N. Educativo padres & $-.125 * *$ & - & & & & & & & & & & \\
\hline 3. N. Socioeconómico & $-.127 * *$ & $.470 * *$ & - & & & & & & & & & \\
\hline 4. Apoyo/Empoderamiento & $-.115^{* *}$ & $.044 *$ & $.093^{* *}$ & - & & & & & & & & \\
\hline 5. Apego al barrio & $-.110 * *$ & $-.059 * *$ & .025 & $.514 * *$ & - & & & & & & & \\
\hline 6. Seguridad & -.065 & $.122 * *$ & $.071 * *$ & $.237 * *$ & $.070 * *$ & - & & & & & & \\
\hline 7. Control social & -.037 & .011 & $.055^{* *}$ & $.343^{* *}$ & $.293 * *$ & $.314^{* *}$ & - & & & & & \\
\hline 8. Actividades & $-.115^{* *}$ & $.049^{*}$ & $.112 * *$ & $.418 * *$ & $.403 * *$ & -.006 & $.188 * *$ & - & & & & \\
\hline 9. P. Externalizantes & $.090 * *$ & $-.086 * *$ & -.008 & $-.137 * *$ & -.011 & $-.201 * *$ & $-.052^{*}$ & -.024 & - & & & \\
\hline 10. P. Internalizantes & $.073^{* *}$ & $-.068 * *$ & $-.095 * *$ & $-.120^{* *}$ & $-.148^{* *}$ & $-.105^{* *}$ & $-.074 * *$ & $-.101 * *$ & $.450 * *$ & - & & \\
\hline 11. Consumo & $.378 * *$ & -.019 & .016 & $-.133^{* *}$ & -.030 & $-.183^{* *}$ & -.028 & $-.045^{*}$ & $.335^{* *}$ & $.080^{* *}$ & - & \\
\hline 12. Satisfacción vital & $-.066^{* *}$ & $.111 * *$ & $.160^{* *}$ & $.218^{* *}$ & $.213^{* *}$ & $.143^{* *}$ & $.130 * *$ & $.136^{* *}$ & $-.213^{* *}$ & $-.402 * *$ & $-.109 * *$ & - \\
\hline
\end{tabular}

$* * p<.01, * p<.05$

con el apoyo/empoderamiento, la seguridad y el control social, en el caso de los problemas externalizantes, y con el apoyo/empoderamiento, la seguridad y las actividades, en el caso del consumo de sustancias. Estas correlaciones tuvieron el sentido esperado, es decir, a puntuaciones más altas en las dimensiones del barrio menos problemas y consumo y más satisfacción vital.

Las variables sociodemográficas incluidas en el estudio también se asociaron de forma significativa con las variables del barrio. Así, se observaron correlaciones negativas significativas entre la edad y la percepción del barrio, de forma que aquellos adolescentes de más edad mostraron percepciones más negativas. También el nivel educativo parental y el socioeconómico familiar guardaron relación con dichas percepciones que eran más favorables según aumentaban ambos niveles, aunque las correlaciones fueron débiles.

Por último, también fueron significativas algunas correlaciones entre las variables sociodemográficas y las referidas al ajuste personal. En términos globales, puede decirse que los problemas internalizantes y externalizantes, incluyendo el consumo de sustancias, aumentaban y la satisfacción vital disminuía, según aumentaba la edad y decrecía el nivel educativo y socioeconómico familiar. Pero al igual que en el caso anterior, las correlaciones fueron de carácter débil, salvo en el caso de la relación entre la edad y el consumo de sustancias.

Cuando se comparó el sexo de los adolescentes con sus puntuaciones en la escala se observaron algunas diferencias significativas, ya que las chicas puntuaron más alto en apoyo/empoderamiento, $F(1,2391)=$

Tabla 2. Regresión múltiple sobre las variables problemas externalizantes y problemas internalizantes

\begin{tabular}{lccc}
\hline Predictores & Beta & $\begin{array}{c}\text { P. Externalizantes } \\
\mathrm{R}^{2} \mathrm{y} \text { sig. del cambio }\end{array}$ & $\begin{array}{c}\text { P. Internalizantes } \\
\mathrm{R}^{2} \mathrm{y} \text { sig. del cambio }\end{array}$ \\
\hline Paso 1 & & \\
\hline Sexo & $.097 * * *$ & $.217 * * *$ \\
Edad & $.087 * * *$ & $.060^{* *}$ \\
N. Educativo padres & $-.094 * * *$ & -.015 & $.060 * * *$ \\
N. Socioeconómico & $.052^{*}$ & $.025^{* * * *}$ & $-.072^{* * *}$ \\
\hline
\end{tabular}

Paso 2

\begin{tabular}{lcl}
\hline Sexo & $.126 * * *$ & $.224 * * *$ \\
Edad & $.073 * * *$ & .038 \\
N. Educativo Padres & $-.067 * *$ & -.015 \\
N. Socioeconómico & $.060 * *$ & $-.061 * *$ \\
Apoyo/Empoderamiento & $-.154 * * *$ & $-.052 *$ \\
Apego al barrio & $.069 * *$ & $-.100^{* * *}$ \\
Seguridad & $-.177 * * *$ & $-.088^{* * *}$ \\
Control Social & .025 & .001 \\
Actividades & .022 & -.006 \\
\hline
\end{tabular}

$* * * p<.001, * * p<.01,{ }^{*} p<.05$ 
Tabla 3. Regresión múltiple sobre las variables consumo de sustancias y satisfacción vital

\begin{tabular}{llll}
\hline Predictores & Beta & $\begin{array}{c}\text { Consumo } \\
\mathrm{R}^{2} \mathrm{y} \text { sig. del cambio }\end{array}$ & $\begin{array}{c}\text { Satisfaccion vital } \\
\mathrm{R}^{2} \mathrm{y} \text { sig. del cambio }\end{array}$ \\
\hline Paso 1 & & & \\
\hline Sexo & .004 & $-.055^{* *}$ \\
Edad & $.388^{* * *}$ & $-.043^{*}$ \\
N. Educativo padres & -.002 & .040 \\
N. Socioeconómico & $.067^{* *}$ & $.135^{* * *}$ \\
\hline Paso 2 & & $.148^{* * *}$ & \\
\hline Sexo & & & $.033^{* * *}$ \\
Edad & .025 & $-.067^{* * *}$ \\
N. Educativo Padres & $.377^{* * *}$ & -.010 \\
N. Socioeconómico & .024 & $.045^{*}$ \\
Apoyo/Empoderamiento & $.073^{* * *}$ & $.114^{* * *}$ \\
Apego al barrio & $-.113^{* * *}$ & $.106^{* * *}$ \\
Seguridad & $.065^{* *}$ & $.140^{* * *}$ \\
Control Social & $-.163^{* * *}$ & $.092^{* * *}$ \\
Actividades & .049 & .017 \\
\hline
\end{tabular}

*** $p<.001, * * p<.01,{ }^{*} p<.05$

$10.63, p<.001$, eta $^{2}=.004 ;$ seguridad, $F(1,2391)=$ $17.69, p<.001, e t a^{2}=.007$ y control social, $F(1$, $2391)=5.92, p=.015$, eta $^{2}=.002$. En cambio, los chicos percibieron más actividades para jóvenes que las chicas, $F(1,2391)=17.97, p<.001$, eta ${ }^{2}=.007$. En apego al barrio no aparecieron diferencias significativas.

Finalmente, y para analizar en mayor profundidad las relaciones entre las variables referidas al barrio y las relativas al ajuste adolescente, se llevaron a cabo regresiones múltiples jerárquicas tanto sobre los problemas internalizantes y externalizantes como sobre el consumo de sustancias y la satisfacción vital (ver Tablas 2 y 3). En un primer paso, y a modo de control, fueron incluidos el sexo, la edad, el nivel educativo parental y el estatus socioeconómico familiar. En un segundo paso se añadieron las cinco dimensiones del barrio o vecindario.

En cuanto a los problemas externalizantes, las variables demográficas introducidas en el primer paso explicaron un $2.5 \%$ de la varianza, indicando que estos problemas eran más frecuentes entre las chicas, y según aumentaban la edad y disminuía el nivel educativo parental. Al añadir en el segundo paso las variables del vecindario, la varianza explicada aumentó de forma significativa hasta un $7.9 \%$. Tanto la mayor seguridad como el mayor apoyo y empoderamiento de la juventud contribuyeron significativamente a explicar la disminución en problemas externalizantes. El mayor apego al barrio mostró una relación positiva paradójica con el aumento de los problemas, aunque el tamaño del efecto de esta relación fue insignificante.

En el caso de los problemas internalizantes, el sexo, la edad y el nivel socioeconómico contribuyeron signi- ficativamente a explicar un $6 \%$ de su varianza, ya que estos problemas fueron más frecuentes entre las chicas, al aumentar la edad y al disminuir el nivel socioeconómico. La inclusión en un segundo paso de las variables referidas al vecindario aumentó de forma significativa este porcentaje hasta un $8.9 \%$, siendo el apego al barrio, la seguridad y el apoyo/empoderamiento las variables que explicaron este incremento de la varianza: los problemas fueron más frecuentes en la medida en que disminuían las puntuaciones en estas tres dimensiones del barrio.

Cuando la variable dependiente fue el consumo de sustancias, las variables sociodemográficas que mostraron una asociación significativa fueron la edad y el nivel socioeconómico familiar: el consumo fue mayor entre los adolescentes de más edad y de mayor nivel socioeconómico. El porcentaje de varianza explicada aumentó significativamente del $14.8 \%$ al $18.6 \%$ al incluir las variables del vecindario, y fueron el apego al barrio, el apoyo/empoderamiento, el control social y la seguridad, las variables que explicaron este aumento, de forma que el consumo fue mayor en sujetos que mostraron puntuaciones más bajas en estas tres últimas tres dimensiones. En cambio, el caso del apego al barrio fueron las puntuaciones más altas las asociadas al mayor consumo, aunque tanto esta asociación como la establecida con el control social, los tamaños del efecto fueron muy bajos.

Finalmente, cuando la variable dependiente fue la satisfacción vital, se observaron relaciones muy parecidas a las anteriores, puesto que de las variables incluidas en el primer paso, el sexo, la edad y el nivel socioeconómico mostraron una relación significativa y explicaron un $3.3 \%$ de la varianza: la satisfacción fue 
mayor entre chicos, de menos edad y de mayor nivel socioeconómico. Al añadir las variables del barrio el porcentaje aumentó significativamente hasta el 9.8\%, y otra vez fueron la vinculación, el apoyo/empoderamiento y la seguridad las variables que mantuvieron una relación significativa con la satisfacción vital. Es decir, los adolescentes que tenían percepciones de mayor apoyo y empoderamiento, más seguridad y que se sentían más vinculados a su barrio fueron quienes también declararon más satisfacción vital.

\section{Discusión}

Los resultados del estudio aportan evidencia sobre la relación entre algunas dimensiones o activos del barrio y el ajuste y la satisfacción vital de los adolescentes que residen en él, tal como habíamos planteado en nuestra hipótesis inicial. El hecho de que esta relación se mantuviese, incluso después de haber controlado estadísticamente algunas variables sociodemográficas, como el nivel de estudios de los padres y el estatus socioeconómico de la familia, añade valor a los resultados, ya que indica que la posible influencia del barrio no se debe exclusivamente al nivel socioeconómico de sus residentes, sino que además existen otras características vecinales o comunitarias que deben ser tenidas en cuenta de cara a favorecer el ajuste y bienestar adolescente. Probablemente, ésa sea la principal aportación de este estudio, la de proporcionar evidencia sobre la importancia que dimensiones comunitarias, que van más allá del nivel socioeconómico o del riesgo global del barrio, tienen no sólo para la prevención de problemas, sino también para promover la satisfacción vital. Este último aspecto también merece ser destacado, ya que la mayor parte de los estudios se han centrado en la conducta antisocial y problemática, $\mathrm{y}$ en menor medida en los trastornos emocionales, siendo muy infrecuente que se consideren indicadores de desarrollo positivo adolescente.

De entre todas las dimensiones comunitarias incluidas en la escala, tres de ellas fueron las que establecieron relaciones significativas con todos los indicadores de ajuste adolescente: el apoyo y empoderamiento de la juventud, el apego al barrio y su seguridad. El control social del comportamiento juvenil solo se asoció muy débilmente con el consumo de sustancias, mientras que la existencia de actividades de ocio para adolescentes no se asoció a ninguno de los indicadores de ajuste adolescente.

El apoyo y empoderamiento supone una percepción positiva de la adolescencia por parte de la población adulta del barrio, que se preocupa por sus jóvenes, los apoyan, los valoran y fomentan su participación. Este empoderamiento ha sido considerado por parte del modelo de desarrollo positivo adolescente un activo comunitario que favorece la participación y promueve el desarrollo de algunas competencias personales como la autoestima, la iniciativa personal y la satisfacción vital (Benson et al., 2006), por lo que está más que justificada la relación encontrada en nuestro estudio con algunos indicadores de ajuste personal. En cuanto al apego o vinculación al barrio también demostró ser un importante activo comunitario, aunque solo para el ajuste interno y la satisfacción vital. En el caso de los problemas externalizantes y el consumo de sustancias, la relación fue positiva, es decir a mayor apego más problemas y mayor consumo, aunque con un bajo tamaño del efecto. Por lo tanto, esta dimensión no pareció tener efectos positivos sobre la reducción de problemas de conducta. Aunque existen escasos estudios que hayan considerado el valor predictivo de la vinculación al vecindario, los estudios que han apuntado la importancia de la vinculación con el centro educativo para el ajuste adolescente nos hacían esperar resultados similares del apego al barrio (Catalano, Haggerty, Oesterle, Fleming y Hawkins, 2004). Por otra parte, como se apuntó en la introducción, esta vinculación al vecindario guarda bastante relación con el sentido de comunidad que suele aparecer asociado a indicadores de salud y ajuste (Cantillon et al., 2003).

La seguridad fue otro activo que mostró su importancia, y probablemente se trate de una de las características vecinales que de forma más clara han mostrado su relación con el ajuste comportamental de jóvenes y adolescentes (Beyers, Loeber, Wikström y Stouthamer-Loeber, 2001; Gracia et al. 2010). No obstante, en nuestro estudio se demostró su importancia no sólo para la prevención de problemas comportamentales, sino también para el ajuste emocional y la satisfacción vital, algo también encontrado en otro estudio (Duncan, 2002). Finalmente, el control social por parte de las personas adultas del vecindario del comportamiento inapropiado por parte de jóvenes y adolescentes se vinculó de forma significativa, aunque muy débil, con un mayor consumo de sustancias. Este dato no apoya la hipótesis de algunos autores de que la implicación activa de los adultos para tratar de regular el comportamiento de sus vecinos más jóvenes puede hacer que ajusten su comportamiento a las normas sociales y así prevenir algunos comportamientos antisociales como el consumo de sustancias (Sampson, Morenoff y Earls, 1999).

La disponibilidad en el barrio de actividades extraescolares de ocio fue la única dimensión que no guardó ninguna relación con el ajuste adolescente, lo que resultó algo inesperado, ya que existe una abundante evidencia empírica acerca del importante papel que desempeña la participación en este tipo de actividades para la promoción de la competencia y el ajuste de chicos y chicas (Broh, 2002; Gardner et al., 2008; Mahoney, Harris y Eccles, 2006). Por supuesto, el que en nuestro estudio no se hayan encontrado relaciones significativas, no niega la importancia para el desarrollo adolescente de la participación en actividades de ocio y tiempo libre, ya que no se evaluó la participa- 
ción sino sólo la disponibilidad de las mismas. Nuestros datos pueden sugerir la necesidad de hacer un esfuerzo por intentar conseguir una mayor implicación de los jóvenes en este tipo de actividades, ya que la disponibilidad no garantiza por sí sola la participación.

Aunque con frecuencia se ha considerado que la influencia del estatus socioeconómico del vecindario sobre el desarrollo de niños y adolescentes está mediada por el estilo educativo parental, especialmente porque en barrios desfavorecidos se observa una menor monitorización parental (Beyers et al., 2001; Cantillon, 2006; Simons, Johnson, Beaman, Conger y Whitbeck, 1997), nuestros datos indican que existen variables extrafamiliares que también tienen su importancia a la hora de explicar esta asociación. De hecho, algunos estudios no han podido demostrar que la relación entre residir en vecindarios de riesgo y los problemas de conducta adolescente estén mediados por las prácticas educativas parentales (Gracia et al., 2010; Schonberg y Shaw, 2007).

No obstante, el hecho de que el nivel socioeconómico familiar mantuviese su relación significativa con los cuatro indicadores de ajuste tras la inclusión en las regresiones de las variables referidas al barrio, pone de relieve que dicho nivel no ejerce su influencia sólo a través de dichas variables por lo que deben existir otras variables, tales como los recursos disponibles en la familia o el centro educativo que pueden también mediar en la relación entre el estatus socioeconómico y el ajuste y el bienestar adolescente.

Otro resultado de nuestro estudio que merece la pena destacar es la relación encontrada entre algunas variables sociodemográficas y la percepción del barrio. Así, se encontraron mejores percepciones entre las chicas, entre los adolescentes de menor edad, y entre quienes tenían padres con un mayor nivel educativo y socioeconómico. Esta última relación coincide con los resultados de otros estudios (Leventhal et al., 2009), y ponen de relieve que los vecindarios donde residen familias con mayor nivel socioeconómico suelen ofrecer un mejor contexto de desarrollo para niños y adolescentes. Menos esperables eran las otras dos relaciones, que parecen indicar que son los adolescentes varones de más edad quienes encuentran menos recursos y oportunidades en el barrio en que residen, algo que debería ser tenido en cuenta de cara a la prevención de los problemas de ajuste adolescente.

Como comentamos en la introducción, el segundo objetivo de este estudio era aportar evidencias de validez externa de la Escala para la Evaluación de los Activos del Barrio, cuya estructura dimensional ya había sido validada en otro lugar (Oliva et al., en prensa). Los resultados encontrados nos permiten afirmar que, tal como se había hipotetizado, se encontraron relaciones significativas entre las dimensiones de la escala y los indicadores de ajuste y bienestar adolescente, lo que supone un apoyo a su validación externa.
Los resultados de nuestro estudio tienen algunas implicaciones prácticas de cara a la intervención. Por una parte ponen de manifiesto la importancia del empoderamiento y la valoración positiva de la adolescencia por parte de los residentes adultos. En este sentido, pensamos que la valoración negativa y estereotipada que existe sobre esta etapa evolutiva es un claro obstáculo para dicho empoderamiento, y debería ser combatida mediante campañas que presenten una imagen más normalizada. Ello debería combinarse con un incremento de las vías de participación de la población joven en la vida del barrio, lo que podría conseguirse ofreciéndoles la posibilidad de gestionar espacios y organizar actividades de ocio de acuerdo con sus intereses y necesidades. De esa manera, se podría también conseguir una mayor participación en dichas actividades, ya que con frecuencia éstas son propuestas por los adultos sin tener en cuenta las verdaderas necesidades de las chicas y chicos en cuanto a contenidos, espacios y horarios de las mismas.

Por otra parte, aunque existe un gran apoyo empírico a la importancia para el desarrollo adolescente de la participación en estas actividades, es necesario destacar que para que estas actividades sean realmente promotoras de competencias, deben reunir una serie de características, como tener continuidad en el tiempo, contar con monitores preparados, proporcionar una estructura predecible con normas y rutinas claras, facilitar la creación de relaciones afectuosas positivas y redes de apoyo o aportar experiencias relevantes y desafiantes para los jóvenes (Hall, Yohalem, Tolman y Wilson, 2002; Parra, Oliva y Antolín, 2009). La seguridad del barrio ha sido otro factor que ha mostrado su importancia, por lo que promover un entorno seguro en el que los adultos se impliquen activamente en la supervisión del comportamiento juvenil, y en el que el vandalismo o la venta y consumo de drogas estén ausentes, debe ser un objetivo prioritario de cara a la prevención comunitaria de problemas de conducta adolescente. Además, debe tenerse en cuenta que un vecindario en el que chicos y chicas se sientan seguros, y que les ofrezca oportunidades para participar y alternativas para el empleo del tiempo libre, facilitará el sentimiento de pertenencia y apego al barrio, algo que este estudio ha revelado como un importante activo comunitario.

Para terminar, nos gustaría hacer referencia a algunas de las limitaciones de este estudio, como su carácter transversal, que imposibilita el establecimiento de relaciones de causalidad entre las dimensiones del barrio y el ajuste adolescente, o el bajo tamaño del efecto de algunas de las relaciones encontradas. También es necesario hacer referencia a la utilización del adolescente como única fuente de información, que ha podido aumentar las correlaciones entre las variables estudiadas. No obstante, consideramos que a pesar de esas limitaciones, los resultados de este estudio contribuyen a un mejor conocimiento de la relación entre algunas de 
las características del barrio y el ajuste y satisfacción de los adolescentes que residen en él, lo que resulta de vital importancia para la práctica de la intervención psicosocial.

\section{Agradecimientos}

Este estudio ha sido realizado gracias a la financiación proporcionada a los autores por la Consejería de Salud de la Junta de Andalucía.

\section{Referencias}

Achenbach, T. M. (1991). Manual for the Youth Self Report and 1991 profile. Burlington, VT, USA: University of Vermont.

Benson, P. L., Scales, P. C., Hamilton, S. F. y Sesman, A. (2006). Positive youth development: Theory, research and applications. En R.M. Lerner (Ed.), Theoretical models of human development. Volume 1 of Handbook of Child Psychology 6th ed., 894-941. Editors-in-chief: W. Damon and R. M. Lerner. Hoboken, New Jersey: Wiley.

Beyers, J. M., Loeber, R., Wikström, P. H. y StouthamerLoeber, M. (2001). What predicts adolescent violence in better-off neighborhoods? Journal of Abnormal Child Psychology, 29, 369-338.

Boyce, W., Torsheim, T., Currie, C. y Zambon, A. (2006). The family affluence scale as measure of national wealth: validation of adolescent self-report measure. Social Indicators Research, 78, 473-487.

Boyle, M. H., Georgiades, K., Racine, Y. y Mustard, C. (2007). Neighborhood and family influences on educational attainment: Results from the Ontario Child Health Study Follow-up 2001. Child Development, 78, 168-189.

Brody, G. H., Ge, X., Conger, R., Gibbons, F. X., Murry, V. M., Gerrard, M., Simons, R. L. (2001). The influence of neighborhood disadvantage, collective socialization, and parenting on African American children's affiliation with deviant peers. Child Development, 72,1231-1246.

Brofenbrenner, U. (1979). The Ecology of Human Development. Cambridge, MA: Harvard University Press.

Broh, B. A. (2002). Linking extracurricular programming to academic achievement: Who benefits and why? Sociology of Education, 75, 69-95.

Burton, L. M. y Jarret, R. L. (2000). In the mix, yet on the margins: The place of families in urban neighborhood and child development research. Journal of Marriage and Family, 62, 1114-1135.

Calafat, A., Mantecón, A., Juan, M., Adrover-Roig, D., Blay, N. y Rosal, F. (2011). Violent behaviour, drunkenness, drug use, and social capital in nigthtlife contexts. Psychosocial Intervention, 20, 45-51.

Cancino, J. M. (2005). The utility of social capital and collective efficacy: social control policy in nonmetropolitan settings. Criminal Justice Policy Review, 16, 287318.

Cantillon, D. (2006). Comunity social organization, parents, and peers as mediators of perceived neighborhood block characteristics on delinquent and prosocial activities. American Journal of Community Psychology, 37, 111127.

Cantillon, D., Davidson, W. S. y Schweitzer, J. H. (2003). Measuring community social organization: Sense of community as a mediator in social disorganization theory. Journal of Criminal Justice, 31, 321-339.

Catalano, R. F., Haggerty, K. P., Oesterle, S., Fleming, C. B. y Hawkins, J. D. (2004). The importance of bonding to school for healthy development: Findings from the Social Development Research Group. Journal of School Health, 74, 252-261.

Chipuer, H. M. y Pretty, G. H. (1999). A review of the Sense of Community Index: Current uses, factor structure, reliability and further development. Journal of Community Psychology, 27, 643-658.

Cleveland, H. H. y Gilson, M. (2004). The Increased Importance of Mother-child Relationships on Sexual Behaviors of Adolescents in Disadvantaged Neighborhoods. Journal of Youth and Adolescence, 33, 319-329.

Cohen, S., Gottlieb, B. y Underwood, L. (2000). Social relationships and health. En S. Cohen, L. Underwood, \& B. Gottlieb (Eds.), Social support measurement and intervention: A guide for health and social scientists (pp. 325). Oxford: Oxford University Press.

Duncan, S. C., Duncan, T. E. y Strycker, L. A. (2002). A Multilevel Analysis of Neighborhood Context and Youth Alcohol and Drug Problems. Prevention Science, 3, 125134.

Dupéré, V., Lacourse, E., Willms, J. D., Leventhal, T. y Tremblay, R. E. (2008). Neighborhood poverty and early transition to sexual activity in young adolescents: a developmental ecological approach. Child Development, 79, 1463-1476.

Eccles, J. S. y Gootman, J. A. (Eds.) (2002). Community programs to promote youth development. Washington, DC: National Academy Press.

Galíndez, E. y Casas, F. (2010). Adaptación y validación de la Students' Life Satisfaction Scale (SLSS) con adolescentes. Estudios de Psicología, 31, 79-88.

Gardner, M., Roth, J. y Brooks-Gunn, J. (2008). Adolescents' participation in organized activities and developmental success two and eight years after high school: Do sponsorship, duration, and intensity matter? Developmental Psychology, 44, 814-830.

Ge, X., Brody, G. H., Conger, R. D., Simons, R. L. y Murry, V. (2002). Contextual amplification of the effects of pubertal transition on African American children's deviant peer affiliation and externalized behavioral problems. Developmental Psychology, 38, 42-54.

Gracia, E. y Herrero, J. (2004). Personal and situational determinants of relationship-specific perceptions of social support. Social Behavior and Personality, 32, 459476.

Gracia, E., Fuentes, M. C. y García, F. (2010). Barrios de riesgo, estilos de socialización parental y problemas de 
conductas en adolescentes. Intervención Social, 19, 265278.

Hall, G., Yohalem, N., Tolman, J. y Wilson, A. (2002). How afterschool programs can most effectively promote positive youth development as a support to academic achievement. National Institute on Out-of-School Time.

Halpern, D. (2005). Social Capital. Cambridge: Polity.

Haynie, D. L., Silver, E. y Teasdale, B. (2006). Neighborhood characteristics, peer influence, and adolescent violence. Journal of Quantitative Criminology, 22, 147-169.

Herrero, J. y Gracia, E. (2007). Measuring perceived community support : Factorial structure, longitudinal invariance, and predictive validity of the PCSQ (Perceived Community Support Questionnaire). Journal of Community Psychology, 35, 197-217.

Huebner, E. S. (1991). Initial development of the Students' Life Satisfaction Scale. School Psychology International, 12, 231-243.

Jencks, C. y Mayer, S. (1990). The social consequences of growing up in a poor neighborhood. En L. E. Lynn y M. G. H. McGeary (Eds), Inner-City Poverty in the United States. Washington, DC: National Academic Press.

Jessor, R. y Jessor, S. (1977). Problem behaviour and psychological development: A longitudinal study of youth. San Diego, CA: Academic Press.

Kroneman, L., Loeber, R. y Hipwell, A. E. (2004). Is neighborhood context differently related to externalizing problems and delinquency for girls compared with boys? Clinical Child and Family Psychology Review, 7, 109122

Lambert, S. F., Brown, T., Phillips, C. M. y Ialongo, N. S. (2004). The relationship between perceptions of neighborhood characteristics and substance use among urban African American adolescents. American Journal of Community Psychology, 34, 205-218.

Larson, R. W., Richards, M. H., Moneta, G., Holmbeck, G. y Duckett, E. (1996). Changes in adolescents' daily interactions with their families from ages 10 to 18 : Disengagement and transformation. Developmental Psychology, 32, 744-754.

Lemos, S., Vallejo, G. y Sandoval, M. (2002). Estructura factorial del Youth Self Report (YSR). Psicothema, 14, 816822 .

Leventhal, T. y Brooks-Gunn, J. (2000).The neighborhoods they live in: The effects of neighborhood residence upon child and adolescent outcomes. Psychological Bulletin, 126, 309-337.

Leventhal, T., Dupéré, V. y Brooks-Gunn, J. (2009). Neighborhood influences on adolescent development. En R. M. Lerner y L. Steinberg (Eds.), Handbook of adolescent psychology (pp. 411-443). New York: John Wiley and Sons.

Mahoney, J. L., Harris, A. L., y Eccles, J. S. (2006). Organized activity participation, positive youth development, and the over-scheduling hypothesis. SRCD Social Policy Report, 20, 1-31.

Mahoney, J. y Stattin, H. (2000). Leisure time activities and adolescent antisocial behavior: The role of structure and social context. Journal of Adolescence, 23, 113-127.

Martinez, M. L., Black, M. y Starr, R. H. (2002). Factorial structure of the perceived neighborhood scale (PNS): A test of longitudinal invariance. Journal of Community Psychology, 30, 23-43.

McMillan, D. W. y Chavis, D. M. (1986). Sense of community: A definition and theory. Journal of Community Psychology, 14, 6-23.

O’Connor, M., Hawkins, M. T., Toumbourou, J. W., Sanson, A., Letcher, P. y Olsson, C. A. (2011). The relationship between social capital and depression during the transition to adulthood. Australian Journal of Psychology, 63, 26-35.

Oliva, A., Antolín, L. y López, A. (en prensa). Development and validation of a scale for the measurement of adolescents' developmental assets in the neighborhood. Social Indicators Research. DOI 10.1007/s11205-011-9822-9.

Oliva, A., Ríos, M., Antolín, L., Parra, A., Hernando, A. y Pertegal, A. (2010). Más allá del déficit: Construyendo un modelo de desarrollo positivo adolescente. Infancia y Aprendizaje, 33, 223-234.

Parra, A., Oliva, A. y Antolín, L. (2009). Los programas extraescolares como recurso para fomentar el desarrollo positivo adolescente. Papeles del Psicólogo, 30, 3-13.

Peterson, N. A., Speer, P. W. y McMillan, D. W. (2007). Validation of a brief sense of community scale: Confirmation of the principal theory of sense of community. Journal of Community Psychology, 36, 61-73.

Plunkett, S. W., Abarca-Mortensen, S., Behnke, A. y Sands, T. (2007). Neighborhood structural qualities, adolescents' perceptions of neighborhoods, and Latino youth development. Hispanic Journal of Behavioral Sciences, 29, 1-16.

Putnam, R. D. (1993). The prosperous community: social capital and public life. The American Prospect, 13, 35-42.

Roosa, M. W., White, R. M., Zeiders, K. H. y Tein, J. Y. (2009). An examination of the role of perceptions in neighborhood research. Journal of Community Psychology, 37, 327-341.

Sampson, R. J. y Groves, W. B. (1989). Community structure and crime: testing social disorganization theory. American Journal of Sociology, 94, 774-802.

Sampson, R. J., Morenoff, D. J. y Earls, F. (1999). Beyond Social Capital: Spatial Dynamics of Collec-tive Efficacy for Children. American Sociological Review, 64, 633-660.

Sampson, R. J., Morenoff, J. D. y Gannon-Rowley, T. (2002). Assessing "neighborhood effects": Social processes and new directions in research. Annual Review of Sociology, 28, 443-478.

Schonberg, M. A. y Shaw, D. S. (2007). Do the predictors of child conduct problems vary by high-and-low-levels of socioeconomic and neighborhood risk? Clinical Child and Family Psychology, 10, 101-136.

Simons, R. L., Johnson, C., Beaman, J., Conger, R. D. y Whitbeck, L. B. (1996). Parent and peer group as mediators of the effect of community structure on adolescent problem behavior. American Journal of Community 
Psychology, 24, 145-171.

Weizman, E. R. y Chen, Y. (2005). Risk modifying effect of social capital on measures of heavy alcohol consumption, alcohol abuse, and secondhand effects: National survey findings. Journal of Epidemiology and Community Health, 59, 303-309.

Wickrama, K. A. S., Merten, M. J. y Elder, G. H. Jr. (2005).
Community influence on precocious transitions to adulthood: Racial differences and mental health consequences. Journal of Community Psychology, 33, 639-653.

Zani, B., Cicognani, E. y Albanesi, C. (2001). Adolescents' sense of community and feeling of unsafety in the urban environment. Journal of Community and Applied Psychology, 11, 475-489.

Manuscrito recibido: 20/06/2011

Revisión recibida: 19/09/2011

Manuscrito aceptado: 23/09/2011 